\title{
Aplicación de dispositivos Kinect para personas en estado de discapacidad
}

\author{
Roberto Ferro Escobar", Arnaldo González $z^{* *}$,Wilson Flórez**, William Ruiz ${ }^{* * * *}$
}

\section{Resumen}

El propósito de este artículo es explicar el empleo de herramientas propias de las TIC para mejorar el rendimiento de personas con algún tipo de discapacidad cognitiva en Colombia, mediante el aprovechamiento de aplicaciones y software basados en videojuegos. Con su ayuda es posible crear alternativas interesantes que se pueden evaluar y emplear en diferentes campos del conocimiento (matemáticas, física y otros) a través de juegos interactivos que hacen que el aprendizaje sea más exitoso y basado en la innovación.

Palabras clave: aplicaciones, discapacidad, Kinect

\begin{abstract}
The purpose of this article is to explain the use of ICT tools to improve the performance of people with some type of cognitive disability in Colombia, through the use of applications and software based on video games. With their help it is possible to create interesting alternatives that can be evaluated and used in different fields of knowledge (mathematics, physics and others) through interactive games that make learning more successful and based on innovation.
\end{abstract}

Keywords: Apps, Disability, Kinect

* Docente investigador del programa de Ingeniería de Sistemas de la Corporación Unificada Nacional de Educación Superior (CUN). Contacto: roberto_ferro@cun.edu.co

** Docente investigador del programa de Ingeniería de Sistemas de la Corporación Unificada Nacional de Educación Superior (CUN). Contacto: arnaldo_gonzalez@cun.edu.co

*** Docente investigador del programa de Ingeniería de Sistemas de la Corporación Unificada Nacional de Educación Superior (CUN). Contacto: wison_florez@cun.edu.co

**** Docente investigador del programa de Ingeniería de Sistemas de la Corporación Unificada Nacional de Educación Superior (CUN). Contacto: william_ruizmar@cun.edu.co 


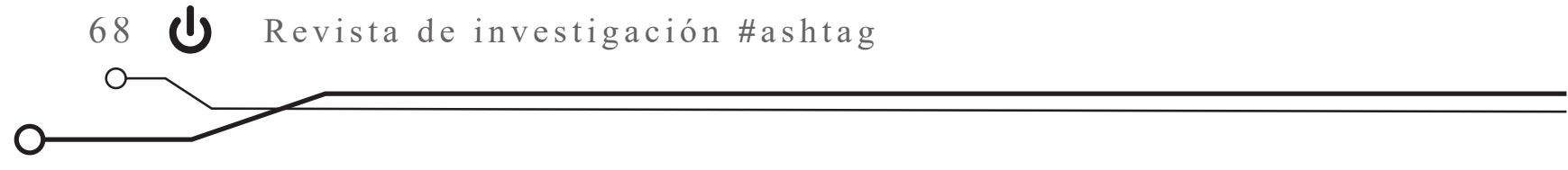

\section{Introducción}

Hoy en día, la innovación se ha convertido en un aspecto clave para la humanidad, pues impulsa el surgimiento de las tecnologías que utilizamos en nuestra vida cotidiana. Actualmente, los desarrollos tecnológicos combinan áreas como la electrónica, la mecánica y la informática, que, en conjunto, logran resolver necesidades humanas de diferentes tipos. Así, pensar en procesos de innovación es una acción crucial para cualquier institución que debe contar con el apoyo de investigadores que promuevan el desarrollo tecnológico de vanguardia. Lo anterior, y en consonancia con las premisas acotadas en la teoría de la educación, permite que estos desarrollos se puedan compartir por medio de redes del conocimiento. Gracias a esto es posible aprender, diseñar y transferir este conocimiento tecnológico a los futuros investigadores, que replicarán estas acciones a los nuevos docentes y estudiantes. Solo de este modo será posible lograr una capacitación integral y erigir el desarrollo tecnológico como la piedra angular de nuestra sociedad.

Después de alcanzar un alto nivel de transferencia de conocimiento en el país, se hace posible pensar en soluciones innovadoras para problemas estructurales y coyunturales de la sociedad, entre ellos, la atención a la población con algún grado de discapacidad -sea de origen genético o debido al conflicto armado que nuestro país ha sufrido durante los últimos cincuenta años-. De acuerdo con las estadísticas publicadas por el Dane (2010), 6 de cada 100 colombianos padecen algún tipo de deterioro cognitivo, lo que habla de un número cercano a las 840000 de personas. De acuerdo con lo anterior, los grupos de investigación aúnan esfuerzos para desarrollar softwares de gamificación capaces de ayudar a mejorar la forma en que estas personas aprenden $y$, por lo tanto, ampliar las alternativas de su inclusión futura en la vida laboral.

\section{Marco legal colombiano sobre discapacidad}

En Colombia, la Fundación para la Investigación en Educación Especial (FIDES) y la Fundación Universitaria de Ciencias de la Salud (FUCS) realizaron un acuerdo de cooperación para la investigación en temas de población en condiciones especiales (Vanguardia, 2 de mayo del 2014). Transformar y mejorar los factores relacionados con la discapacidad, prevenir su aparición y promover el desarrollo potencial de las personas en estado de discapacidad es un tema de salud pública que requiere un esfuerzo de trabajo conjunto entre el Estado, las organizaciones privadas, las organizaciones sociales, las universidades y la sociedad civil. Sin esta condición, no será posible garantizar la realización personal y el desarrollo de la libre participación de todos los miembros de la sociedad en sus dinámicas de trabajo (Forero, 2004).

El marco legal para incluir a las personas con discapacidades en Colombia se inició a partir de la Constitución de 1991, particularmente en el artículo 13: “El Estado protege especialmente a aquellas personas cuyas condiciones económicas, físicas o mentales están en circunstancias obviamente vulnerables y sanciona cualquier abuso o maltrato que se les haya cometido". Por su parte, el artículo 68 estipula que "la 
erradicación del analfabetismo y la educación de las personas con limitaciones físicas o mentales o con capacidades excepcionales son obligaciones especiales del Estado". El artículo 47 también hace una referencia específica a las discapacidades cognitivas al afirmar que "el Estado promoverá una política de planificación, rehabilitación e integración social de personas con discapacidades físicas, sensoriales y mentales, que requieran atención especializada" (Forero, 2004).

En 1981, el Gobierno colombiano emitió el Decreto 2358, que rige la rehabilitación profesional y el empleo de personas discapacitadas. En 1989, sancionó el Decreto 2177, el Decreto Ley 2737 y el Código de Menores. En cuanto a la historia de la formulación de planes para la "Política de prevención y discapacidad", cuyos principales objetivos fueron mejorar la calidad de vida de la población en general y el logro de su integración social y económica, son de recordar algunos hitos: en 1995 se emitió el documento Conpes 2761, relacionado con temas de discapacidad; en 1997, el Gobierno reglamentó la Ley 361 de 1997, “por la cual se establecen mecanismos de integración social de personas con limitaciones". Existen también mecanismos de prevención, educación, rehabilitación, integración laboral, bienestar social y accesibilidad. Para el ámbito deportivo, se promulgó la Ley 181 de 1995, que dicta reglas para la promoción del deporte asociado a personas con discapacidades cognitivas y medidas orientadoras para su rehabilitación e integración social. El Gobierno también estableció el "Plan nacional de atención a personas con discapacidad" entre 1999 y 2002, las "Bases para el Desarrollo de una Política Pública de Discapacidad 2003-2006", y presentó al Consejo Presidencial para la Política Social como la organización responsable de la coordinación de las actividades de interacción entre las diferentes instituciones nacionales involucradas. Ahora, al revisar el estado del arte sobre asuntos legales, se puede ver que existe una amplia gama de artículos y leyes sobre el tema, pero estas no parecen repercutir positivamente en el mejoramiento de la situación real de las personas con discapacidad: su marginación de los espacios culturales, educativos y laborales es evidente, sin contar con la que sufren en el marco de los procesos políticos y sociales de la nación.

\section{Estado del arte en temas internacionales sobre personas con discapacidad}

Las Naciones Unidas han promulgado diferentes declaraciones, convenciones, planes $\mathrm{y}$ varios documentos con recomendaciones que contienen algunas propuestas relacionadas con los derechos de las personas con discapacidad. Allí se identifican los deberes de los Estados y de la sociedad hacia ellos, y se elaboran pautas de acción para prevenir la discapacidad, proporcionar atención, crear condiciones para la integración social y superar cualquier forma de discriminación (Forero, 2004):

- Convención Americana sobre Derechos Humanos: se ocupa de las medidas para proteger a los menores y la igualdad ante la ley.

- Convenio 159 sobre rehabilitación profesional y empleo de personas con discapacidad: adicional al Protocolo de la Convención 


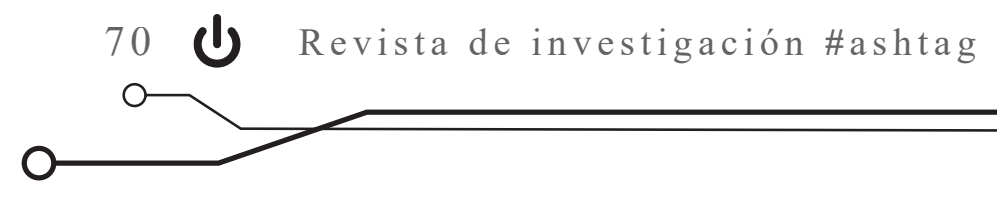

Americana sobre Derechos Humanos, Económicos, Sociales y Culturales.

- Protocolo de San Salvador: los Estados se comprometen a adoptar, de conformidad con sus procedimientos constitucionales, las medidas legislativas $\mathrm{u}$ otras que sean necesarias para hacer efectivos el derecho de no discriminación para las poblaciones propensas a algún tipo de violación.

- Convención de los Derechos del Niño: establece que un niño con discapacidad mental o física debe disfrutar de una vida plena y digna, en condiciones que garanticen la dignidad y la autosuficiencia y faciliten su participación activa en la comunidad.

- Convención Americana sobre la Eliminación de todas las Formas de Discriminación contra las Personas con Discapacidad.
- Declaración de los Derechos del Retrasado Mental.

- Programa de Acción Global para Personas con Discapacidad.

- Declaración y Programa de Acción de Viena: acerca de la situación de las personas con discapacidad en las Américas.

- Compromiso de Panamá con las personas con discapacidad en las Américas (OEA).

- Reglas estándar de la ONU sobre la igualdad de oportunidades para las personas con discapacidad.

- Derechos humanos de las personas con discapacidad.

\section{Discapacidad de aprendizaje y otras áreas}

La frase "discapacidad específica de aprendizaje" significa el trastorno en uno o más procesos psicológicos básicos involucrados en la comprensión o en el uso del lenguaje (hablado o escrito), que puede manifestarse en una capacidad imperfecta para escuchar, hablar, leer, escribir, deletrear o para hacer cálculos matemáticos. El término incluye condiciones tales como discapacidades perceptivas, lesión cerebral, disfunción cerebral mínima, dislexia y afasia del desarrollo. En todo caso, no se aplica a los niños que tienen problemas de aprendizaje cuando son resultado de discapacidades visuales, auditivas o motoras, de retraso mental, de trastornos emocionales o de desventajas ambientales, culturales o económicas (Cornell, 2006). Según la publicación de Reid, la discapacidad de aprendizaje es una categoría general de educación especial compuesta por discapacidades en cualquiera de las siete áreas específicas (Reid, 1996): lenguaje receptivo (escuchar), lenguaje expresivo (habla), habilidades básicas de lectura, comprensión lectora, expresión escrita, cálculo matemático y razonamiento matemático.

Estos tipos separados de discapacidades de aprendizaje suelen coexistir entre sí y también con ciertos déficits de habilidades sociales y trastornos emocionales o de comportamiento, como el trastorno por déficit de atención. La discapacidad de aprendizaje no es sinónimo de discapacidad de lectura o dislexia, aunque a menudo se malinterpreta como tal; sin embargo, la mayor parte de la información disponible sobre 
discapacidades de aprendizaje se relaciona con las discapacidades de lectura, y la mayoría de los niños con discapacidades de aprendizaje tienen sus principales deficiencias en la lectura. Ahora, de acuerdo con las definiciones anteriores y la publicación estadística realizada por el Dane (2010) sobre personas con discapacidad en Colombia, es posible distinguir varias categorías o tipos de alteración o enfermedad específica de acuerdo a su ubicación en el cuerpo. En la tabla 1 se presentan los datos recopilados en la ciudad de Bogotá, una muestra muy representativa en nuestro país.

Tabla 1. Datos de personas con algún tipo de discapacidad en Bogotá

\begin{tabular}{|c|c|c|c|c|c|c|c|c|c|c|c|c|}
\hline \multirow[b]{2}{*}{ 解 } & \multicolumn{12}{|c|}{ ALTERACIÓN } \\
\hline & 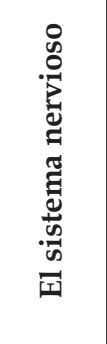 & $\begin{array}{l}\stackrel{0}{0} \\
\stackrel{0}{0} \\
0 \\
0 \\
0\end{array}$ & $\begin{array}{l}n \\
0 \\
0 \\
0 \\
0 \\
0 \\
0 \\
0\end{array}$ & 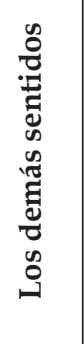 & 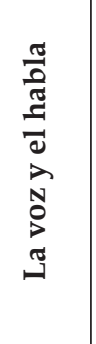 & 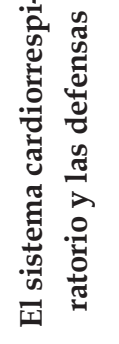 & 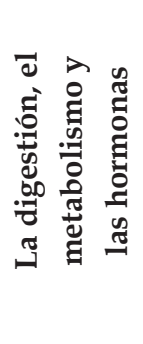 & 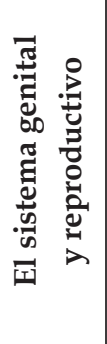 & 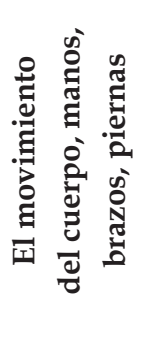 & 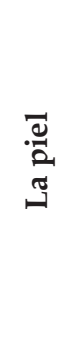 & $\stackrel{\pi}{0}$ & సేّ \\
\hline $\begin{array}{l}11101 \\
\text { Usaquén }\end{array}$ & 3092 & 2693 & 960 & 188 & 886 & 5689 & 3107 & 828 & 4621 & 345 & 1093 & 23502 \\
\hline $\begin{array}{l}11102 \\
\text { Chapinero }\end{array}$ & 1827 & 665 & 312 & 40 & 361 & 1133 & 669 & 276 & 902 & 131 & 92 & 6408 \\
\hline $\begin{array}{l}11103 \\
\text { Santafé }\end{array}$ & 1512 & 1235 & 489 & 109 & 472 & 995 & 404 & 282 & 2021 & 134 & 170 & 7823 \\
\hline $\begin{array}{l}11104 \text { San } \\
\text { Cristóbal }\end{array}$ & 5972 & 4458 & 1750 & 401 & 1845 & 5101 & 2744 & 1598 & 7083 & 445 & 990 & 32387 \\
\hline $\begin{array}{l}11105 \\
\text { Usme }\end{array}$ & 3607 & 2952 & 1496 & 334 & 1563 & 3453 & 1658 & 699 & 4994 & 515 & 66 & 21337 \\
\hline $\begin{array}{l}11106 \\
\text { Tunjuelito }\end{array}$ & 3485 & 3445 & 1812 & 214 & 1006 & 3239 & 1913 & 1026 & 4769 & 343 & 77 & 21329 \\
\hline $\begin{array}{l}11107 \\
\text { Bosa }\end{array}$ & 5820 & 8993 & 3112 & 902 & 1456 & 9945 & 6588 & 2231 & 8520 & 1259 & 251 & 49077 \\
\hline $\begin{array}{l}11108 \\
\text { Kennedy }\end{array}$ & 10671 & 11230 & 3889 & 791 & 2812 & 16023 & 8358 & 2386 & 14380 & 1402 & 1479 & 73421 \\
\hline $\begin{array}{l}11109 \\
\text { Fontibón }\end{array}$ & 2535 & 4140 & 1582 & 302 & 1256 & 4352 & 2610 & 1366 & 4971 & 802 & 393 & 24309 \\
\hline $\begin{array}{l}11110 \\
\text { Engativá }\end{array}$ & 6530 & 4542 & 2177 & 379 & 2582 & 3593 & 2319 & 1216 & 7068 & 559 & 888 & 31853 \\
\hline $\begin{array}{l}11111 \\
\text { Suba }\end{array}$ & 5308 & 4608 & 1998 & 332 & 2378 & 3878 & 1990 & 965 & 7406 & 564 & 445 & 29872 \\
\hline
\end{tabular}




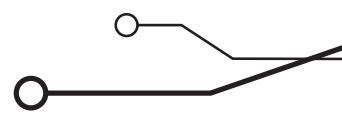

\begin{tabular}{|c|c|c|c|c|c|c|c|c|c|c|c|c|}
\hline $\begin{array}{l}11112 \\
\text { Barrios } \\
\text { Unidos }\end{array}$ & 3528 & 1775 & 771 & 140 & 715 & 2262 & 1395 & 554 & 2302 & 198 & 393 & 14033 \\
\hline $\begin{array}{l}11113 \\
\text { Teusaquillo }\end{array}$ & 1625 & 381 & 246 & 22 & 256 & 784 & 655 & 181 & 734 & 256 & 104 & 5244 \\
\hline $\begin{array}{l}11114 \\
\text { Mártires }\end{array}$ & 1273 & 758 & 360 & 155 & 497 & 896 & 438 & 319 & 1683 & 139 & 45 & 6563 \\
\hline $\begin{array}{l}11115 \\
\text { Antonio } \\
\text { Nariño }\end{array}$ & 1469 & 1625 & 586 & 89 & 371 & 1611 & 801 & 427 & 2090 & 177 & 344 & 9590 \\
\hline $\begin{array}{l}11116 \\
\text { Puente } \\
\text { Aranda }\end{array}$ & 3798 & 4825 & 1701 & 346 & 777 & 6231 & 4048 & 1149 & 6015 & 508 & 575 & 29973 \\
\hline $\begin{array}{l}11117 \\
\text { Candelaria }\end{array}$ & 630 & 569 & 236 & 47 & 100 & 548 & 299 & 213 & 785 & 83 & 19 & 3529 \\
\hline $\begin{array}{l}11118 \text { Ra- } \\
\text { fael Uribe }\end{array}$ & 7280 & 9044 & 3117 & 363 & 1839 & 10692 & 4759 & 1995 & 12154 & 838 & 1178 & 53259 \\
\hline $\begin{array}{l}11119 \\
\text { Ciudad } \\
\text { Bolívar }\end{array}$ & 6068 & 5066 & 2149 & 414 & 2826 & 4076 & 2648 & 1751 & 7005 & 711 & 228 & 32942 \\
\hline $\begin{array}{l}11120 \\
\text { Sumapaz }\end{array}$ & 123 & 59 & 41 & 0 & 31 & 59 & 27 & 4 & 147 & 8 & 41 & 540 \\
\hline Total & 76153 & 73063 & 28784 & 5568 & 24029 & 84560 & 47430 & 19466 & 99650 & 9417 & 8871 & 476991 \\
\hline
\end{tabular}

Fuente: Dane (2010)

De la tabla 1 se puede apreciar que las 5 primeras áreas de personas que presentan discapacidades en orden de mayor a menor son: movimiento, el sistema cardio-respiratorio, el sistema nervioso, ojos y oídos. Con base en lo anterior, se realiza el presente capítulo de investigación, fundamentado en los desarrollos de los grupos de investigación y su grado de responsabilidad social ante esta problemática nacional. Con el ánimo de contribuir en la materia, indagamos por nuevas tecnologías y aplicaciones basadas en el dispositivo Kinect y comenzamos a estudiarlo con el fin de lograr diseños innovadores y prácticos basados en las Tic y de posible aplicación en nuestro país.

\section{Desarrollo de soluciones integrales basadas en tecnología Kinect}

El objetivo principal de este proyecto es desarrollar soluciones integrales que usen la tecnología Kinect (McCutcheon et al., 2014) y el software de aplicación de TIC que permite que personas con algún tipo de discapacidad puedan mejorar su vida diaria o ser incluidos en el campo laboral. 
Estos problemas pueden ser de cualquier tipo y es necesario comenzar a identificar los inconvenientes que se presentan: por ejemplo, movimientos simples (centro, izquierda o derecha) pueden resolverse a través de sonidos diferenciadores (silbidos, voces o indicaciones). Para mejorar el aprendizaje de las personas con discapacidades cognitivas, también se formuló el posible desarrollo de otras aplicaciones basadas en software Scratch, que permiten aprender mediante el uso de juegos interactivos. En cuanto a discapacidad de movimiento, se pueden crear nuevas alternativas de terapias de rehabilitación capaces de insertar laboralmente a personas con cuadriplejia. Los resultados de esta investigación tenderán a crear aplicaciones y software reales basados en el dispositivo Kinect.

\section{Tecnología de identificación}

En esta etapa se realizó una revisión del estado actual del sistema Kinect y se diferenciaron los dispositivos Kinect desarrollados por Microsoft. La primera versión que se lanzó al mercado en el año 2010 fue la Xbox 360. Este dispositivo reconoce completamente los movimientos y permite la interacción con los comandos de sonido y voz. Requiere un amplio espacio para identificar movimientos y elementos, con compatibilidad limitada con el desarrollo de software.

La última versión del dispositivo Kinect que salió al mercado fue a finales de 2014 con la
La creación y generación de proyectos de investigación contribuye al cumplimiento de la misión y los factores académicos e investigativos de las instituciones de educación superior y los centros de investigación, así como a lograr una alta cohesión entre profesores, estudiantes y la población a la que se quiere contribuir. Este proyecto genera nuevos productos de conocimiento que permiten posicionar a los grupos de investigación y sus aliados en el sistema nacional de investigación. Tales productos, a su vez, contribuyen al trabajo social de las instituciones y a mejor comprensión de la responsabilidad social en nuestro país y en el mundo. Los resultados también posicionarán a la institución como entidad capaz de crear nuevos desarrollos y tecnología a nivel nacional e internacional. El proyecto Kinect facilita el intercambio de investigadores y profesores entre diferentes países a nivel internacional.

consola Xbox One, que además de ser mucho más pequeña y portátil, tiene un mayor grado de precisión y velocidad de movimientos y no requiere un espacio tan grande como la versión anterior. Además de lo anterior, el SDK desarrollado por Microsoft permite crear aplicaciones de software, es totalmente compatible con los dispositivos y tiene herramientas que facilitan la maximización de los servicios ofrecidos por Kinect. El sistema Kinect utiliza luz infrarroja y una cámara de video para mapear en 3D el área que hay frente a ella y un algoritmo de búsqueda avanzada (figura 1). 


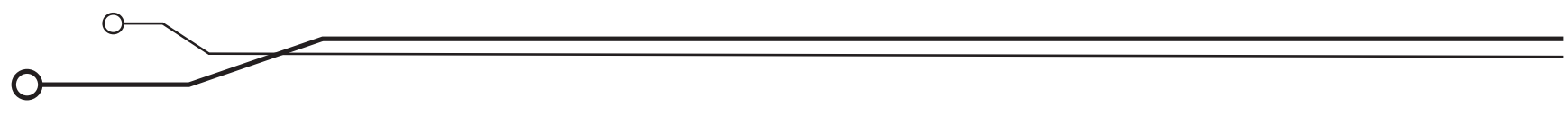

Figura 1. Descripción de Kinect,

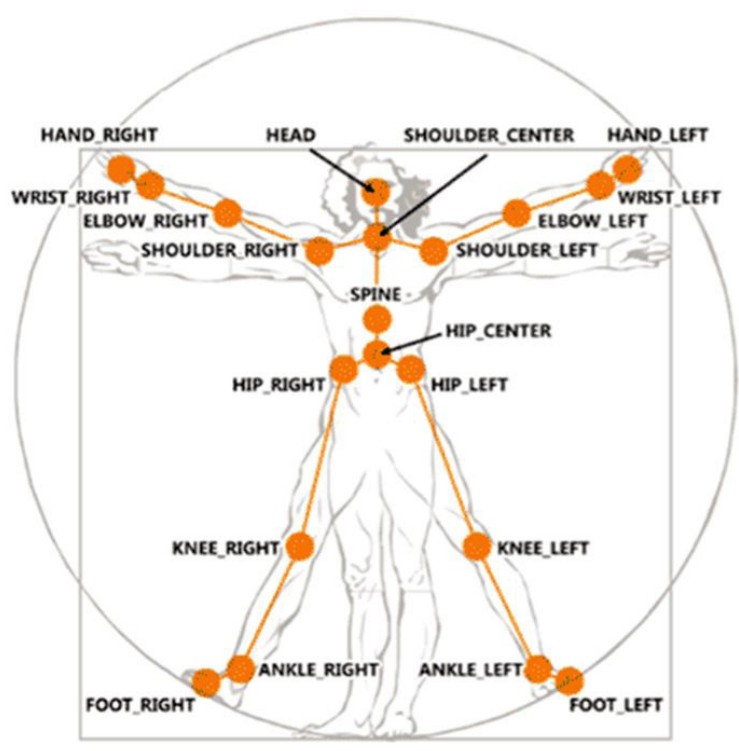

(A)

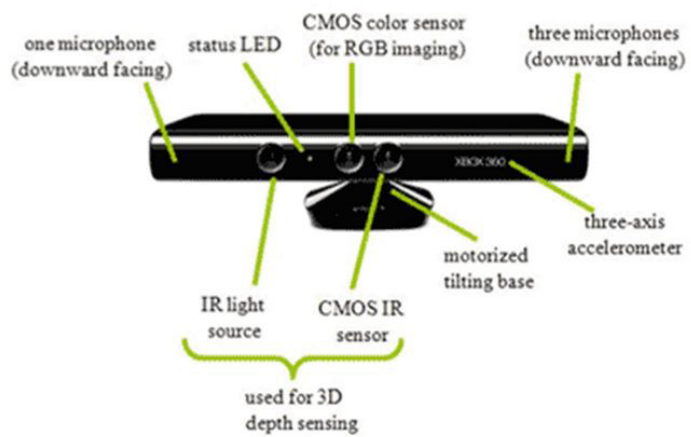

(B)

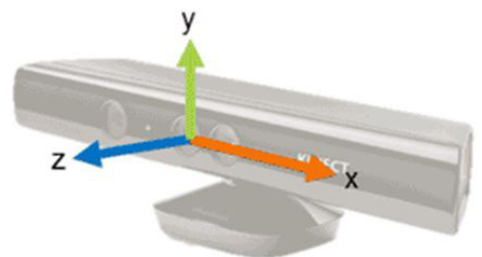

(C)

Fuente: McCutcheon et al. (2014, párr. 3)

\section{Trabajando con SDK API}

En esta etapa se identificó que el dispositivo Kinect para Windows SDK en su versión 1.8 incluye algunas características clave, tales como:

- Eliminación en segundo plano: una API que se descarga e instala y elimina el fondo detrás del usuario activo, por lo que puede ser reemplazado por un fondo artificial diseñado por el usuario. Este efecto de "selección verde" fue una de las primeras solicitudes de los usuarios que realizaron los primeros desarrollos. Este servicio es especialmente útil para publicidad, juegos de realidad aumentada, entrenamiento, simulación y otras experiencias inmersivas que ponen al usuario en un entorno virtual diferente (Clark et al., 2012).

- Captura de colores realistas con Kinect Fusion: el "Kinect Fusion" es otra APr. Esta escanea el color de la escena junto con la información de profundidad para que pueda ser capturado el color del objeto junto con su modelo tridimensional (3D). La API también produce un mapa de textura para la malla creada a partir del escaneo. Esta función proporciona un modelo $3 \mathrm{D}$, incluido el color, que se puede utilizar para imprimir en 3D o para crear actividades 3D, CAD y otras aplicaciones.

- Robustez de rastreo mejorada Kinect Fusion: este algoritmo hace que sea más fácil escanear una escena. Con esta actualización, Kinect Fusion puede mantener su bloqueo en la escena a medida que la cámara se mueve, con lo que produce un escaneo más confiable y consistente. 
- Muestra la interacción HTML: este servicio permite la implementación de los botones Kinect habilitados a través de una interfaz de usuario simple y el uso de una eliminación de fondo de flujo en HTML5. Esto permite a los desarrolladores utilizar HTML5 y JavaScript para implementar interfaces de usuario compatibles con Kinect, lo que antes no era posible.
- Multisensor Kinect Fusion: Este servicio permite a los desarrolladores utilizar simultáneamente dos sensores para escanear una persona u objeto desde ambos lados en busca de una posible construcción de un modelo 3D, sin tener que mover el sensor u objeto.

\section{Primer desarrollo de software Hola Kinect}

De acuerdo con la investigación realizada, en esta sección hicimos que los primeros desarrollos se presenten y modifiquen aspectos del

\section{Ejemplo de código en el dispositivo Kinect}

Para comenzar a programar el sensor Kinect, fue necesario investigar en la página web de Microsoft, ya que deben efectuarse las pruebas iniciales y la puesta en marcha del proyecto. Para que el dispositivo funcione es necesario ejecutar el siguiente código proporcionado por Microsoft (Microsoft Corporation, 2015):

Kinect sensor streams are enabled and configured using JSON. You can retrieve the current configuration by calling the getConfig function

sensor.getConfig(function (configData) $\{\ldots\}$ );

The following example demonstrates how to use the postConfig function to enable and configure the Kinect sensor streams. All valid stream names are shown in this example. sistema Kinect y las propuestas de aplicaciones bajo el sistema operativo Windows (Berman, 2 de junio del 2012).

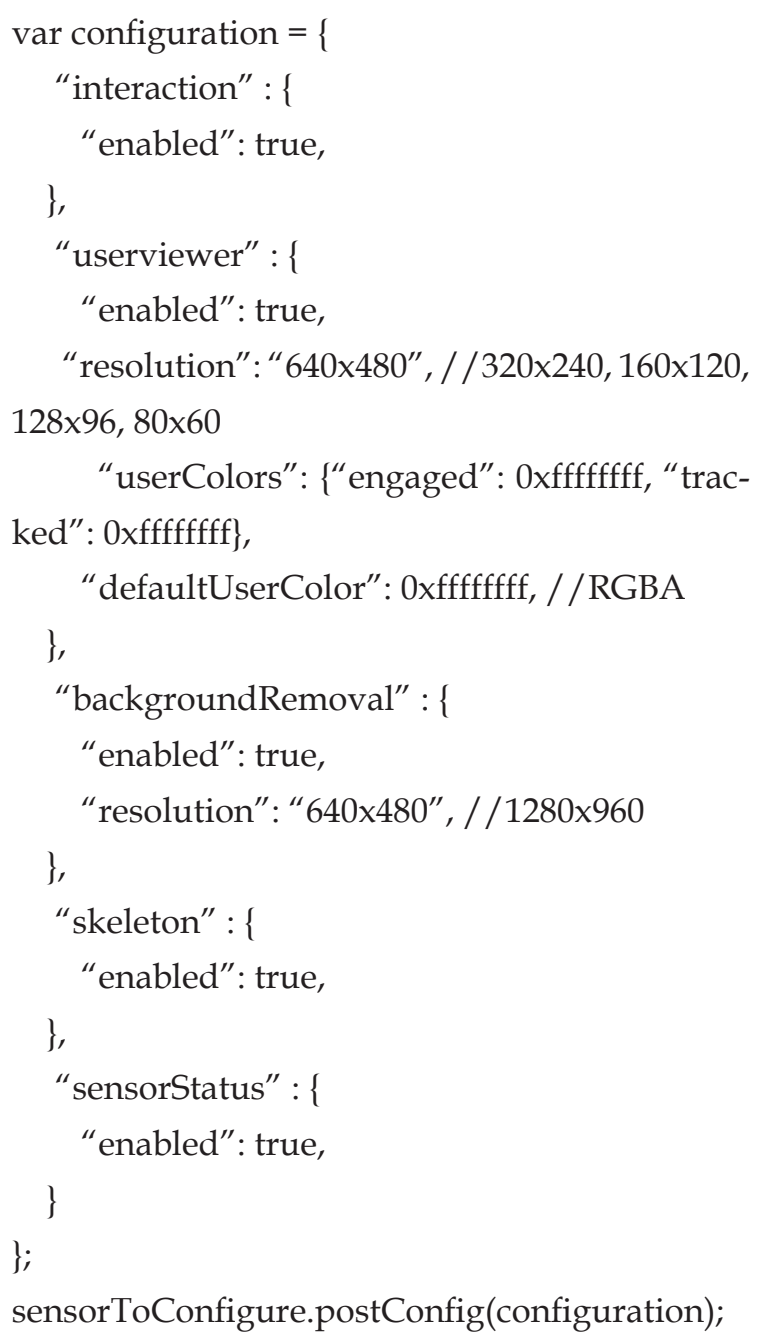




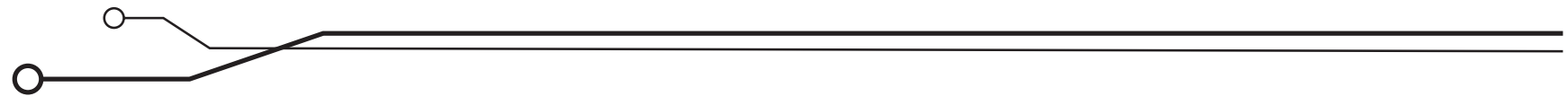

Figura 2. Prueba de inicialización del dispositivo Kinect en laboratorio

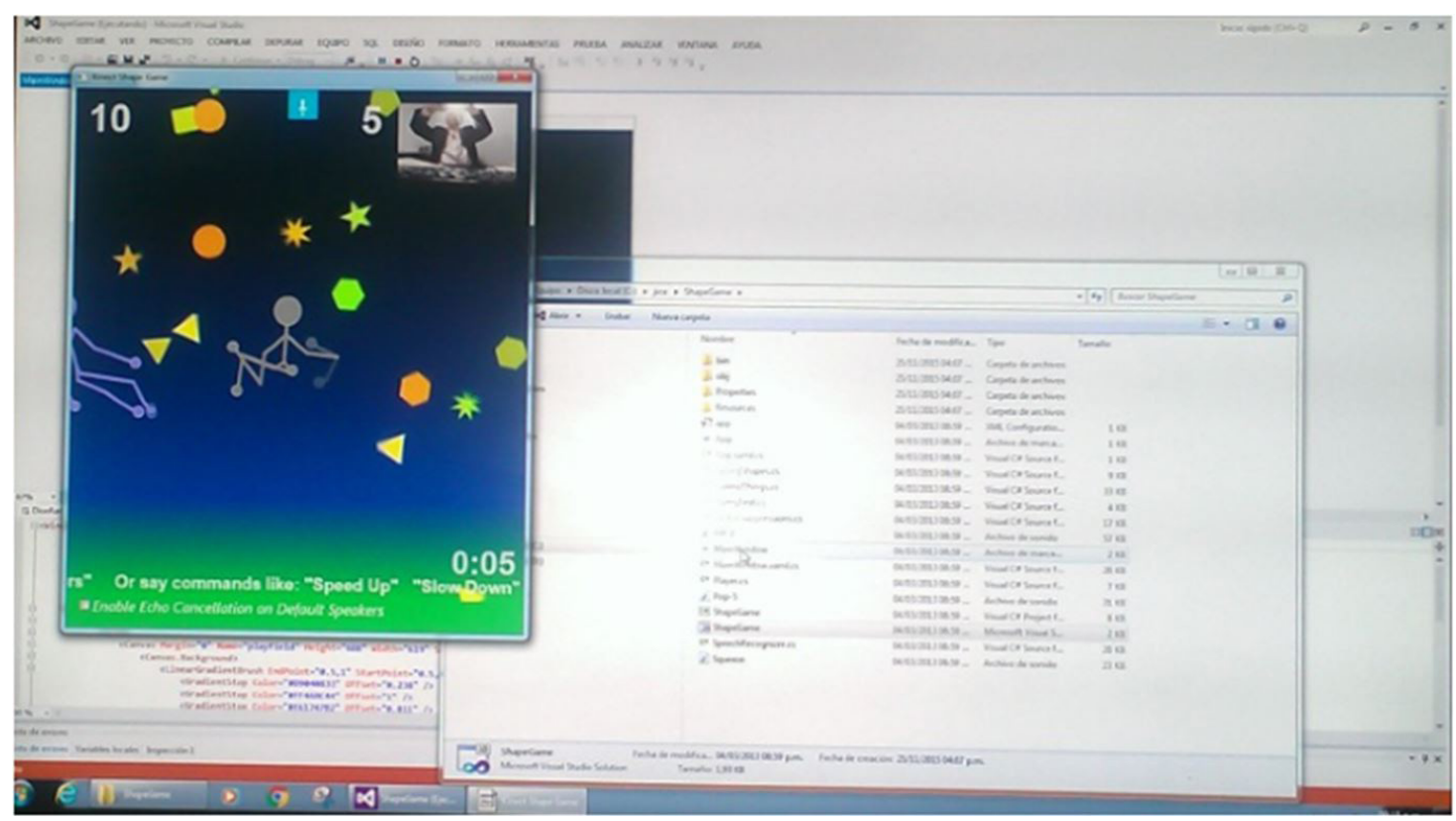

Fuente: elaboración propia

\section{Metodología de desarrollo de juegos basados en Kinect}

Para lograr realizar un juego exitoso usando el dispositivo Kinect es necesario efectuar pruebas de laboratorio y usar una metodología adecuada de diseño de juegos que pueda ser aplicada al e-learning para personas en estado de discapacidad. Las características cuantitativas deseadas son las que se enumeran a continuación:

a) Desarrollo de habilidades de enseñanza-aprendizaje.

b) Autonomía del estado de discapacidad individual.

c) Razonamiento inductivo. d) La creatividad y la innovación.

e) El conocimiento de las disciplinas básicas.

f) Especificación de requisitos (entrada-salida), funcionales, no funcionales y otros.

g) Tipo de juego (estrategia, memoria, agrupación, acuerdo).

La metodología utilizada para el diseño de un juego se basa en la publicación de Luca Galli (2014). El autor establece una serie de pasos para lograr el diseño de un juego para un equipo de desarrolladores formado por programadores, diseñadores gráficos y otros técnicos. 
Figura 3. Proceso de desarrollo de un juego, desde el modelado, las pautas y las mejores prácticas de Fullerton y Galli

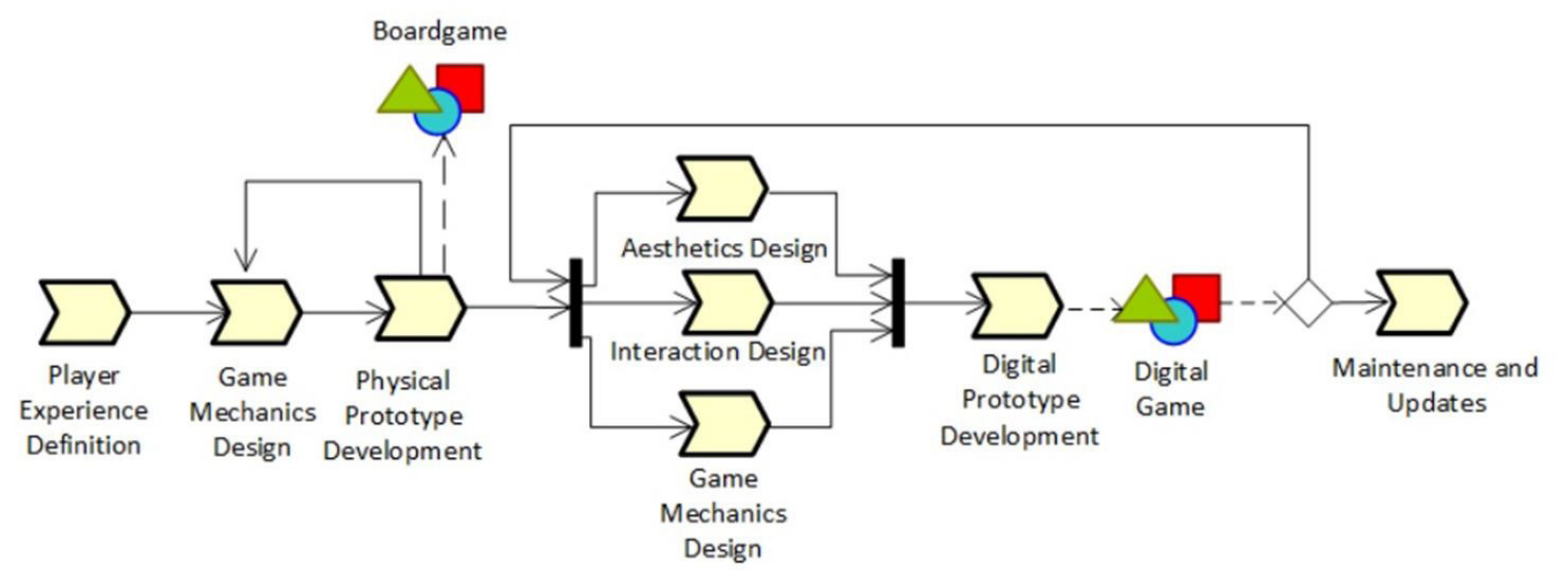

Fuente: Galli (2014, diapositiva 11)

\section{Referencias}

Berman, A. (2 de junio del 2012). Start Coding for the Kinect [entrada de blog]. Microsoft. Recuperaod de https://blogs.msdn.microsoft.com/csharpfaq/2012/02/06/ start-coding-for-the-kinect/

Clark, R., Pua, Y., Fortin, K., Ritchie, C. y Webster, K. (2012). Validity of the Microsoft Kinect for Assessment of Postural Control. Gait and Posture, 36, 372-377. Recuperado de https:/ / www.sciencedirect.com/science/article/abs/pii/S0966636212001282?via\%3Dihub

Cornell Law School. (2006). 34 CFR Part 300. Assistance to States for the Education of Children with Disabilities [recurso en línea]. Recuperado de https://www.law.cornell.edu/cfr/text/34/ part-300

Dane. (2010). Discapacidad [recurso en línea]. Recuperado de https://www.dane.gov.co/index. php/estadisticas-por-tema/demografia-y-poblacion/discapacidad

Forero, A. (2004). Estudio deantecedentes sobreel tema de discapacidad cognitivay su legislación [recurso en línea]. Recuperado de https:/ / vlex.com.co/vid/estudios-antecedentes-senado-451442998

Galli, L. (2014). Matching Game Mechanics and Human Computation Tasks in Games with a Purpose. ACM Workshop on Serious Games, Orlando, Florida, Estados Unidos. Recuperado de http://www.slideshare.net/Leyart1/ matching-game-mechanics-and-human-computation-tasks-in-games-with-a-purpose 


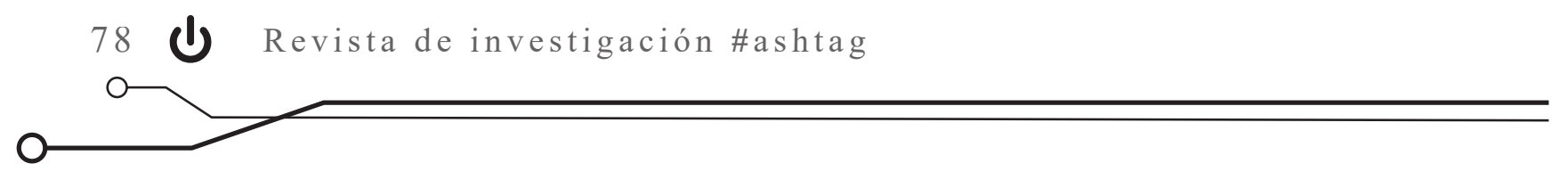

McCutcheon, J, Hwang, S., Ying, C. y Koontz, A. (2014). The Feasibility of Using Kinect for Transfer Assessment. Resna Annual Conference, Indianapolis, Estados Unidos. Recuperado de https://www.resna.org/sites/default/files/conference/2014/Wheelchair\%20Seating/ Student\%20Scientific/McCutcheon.html

Microsoft Corporation. (2015). Human Interface Guidelines v. 1.8 [recurso en línea]. Recuperado de http:/ / go.microsoft.com/fwlink/?LinkID=247735

Reid, G. (1996). Learning Disabilities. The Future of Children, 6(1), 54-76. Recuperado de https:// www.jstor.org/stable/1602494

Vanguardia. (2 de mayo del 2014). 6 de cada 100 colombianos sufren discapacidad física o cognitiva. Recuperado de http://www.vanguardia.com/actualidad/ colombia/258099-6-de-cada-100-colombianos-sufren-discapacidad-fisica-o-cognitiva. 